ESAIM: PROCEEDINGS AND SURVEYS, September 2018, Vol. 61, p. 55-67

Pauline Lafitte and Karim Ramdani, Editors

\title{
FLUCTUATIONS AND TEMPERATURE EFFECTS IN BOSE-EINSTEIN CONDENSATION*
}

\author{
Anne de Bouard ${ }^{1}$, Arnaud Debussche $^{2}$, Reika Fukuizumi $^{3}$ and Romain Poncet ${ }^{4}$
}

\begin{abstract}
The modeling of cold atoms systems has known an increasing interest in the theoretical physics community, after the first experimental realizations of Bose Einstein condensates, some twenty years ago. We here review some analytical and numerical results concerning the influence of fluctuations, either arising from fluctuations of the confining parameters, or due to temperature effects, in the models describing the dynamics of such condensates.

Résumé. Depuis les premières réalisations expérimentales de condensats de Bose-Einstein, il y a maintenant plus de vingt ans, l'étude des sytèmes d'atomes froids a connu une forte croissance dans la communauté des physiciens théoriciens. Nous décrivons ici certains résultats mathématiques et numériques concernant la prise en compte de fluctuations, qui peuvent être dues aux paramètres du laser utilisé pour le confinement des atomes, ou aux effets de la température lorsque celle-ci est proche de la température critique de condensation, dans les modèles régissant la dynamique de ces condensats.
\end{abstract}

\section{INTRODUCTION}

The first experimental realizations of Bose-Einstein condensates of ultra cold gases in 1995 (see $[3,7,11]$ ) have been the starting point of experimental studies of those objects whose behaviour close to superfluidity was predicted a long time ago. But they have also led to a large increase of the theoretical studies, as the comparisons with the experiments were henceforth made feasible.

Mean field techniques have then shown their power in the sense that the Gross-Pitaevskii equation, which is the mean field limit equation for the macroscopic wave function of the condensate, was proven to be able to capture a large part of the physical phenomena for zero-temperature condensates in harmonic traps, especially concerning the energy and density distributions (see [10]). The predictions are in particular in very good agreement with the experiments. An important feature of the system is the role played by the two-body interactions. Indeed, at temperature close to the absolute zero, due to its inhomogeneity, and despite the very dilute nature of the gases, the atom-atom interactions, when taken into account in the model, can lead to density in the center of the condensate much smaller than the density predicted for an ideal gas in the same trap (see [10]). A consequence is that the nonlinearity in the Gross-Pitaevskii equation is essential and cannot be neglected.

* This work was supported by the ANR through project ANR-12-MONU-0007 BECASIM, the LabEx LMH and the JSPS KAKENHI Grant Number 15 K04944.

1 CMAP, Ecole polytechnique, CNRS, Université Paris-Saclay, 91128, Palaiseau, France

2 IRMAR, ENS Rennes, CNRS, UEB. av. Robert Schuman, F-35170 Bruz, France

3 Research Center for Pure and Applied Mathematics, Graduate School of Information Sciences, Tohoku University, Sendai 980-8579, Japan

${ }^{4}$ CMAP, Ecole polytechnique, CNRS, Université Paris-Saclay, 91128, Palaiseau, France

(C) EDP Sciences, SMAI 2018 
When the temperature of a gas is decreased below a critical temperature, the wavelength of the atomic cloud becomes equal to the typical distance between the particles, wich leads to the necessity to take account of quantum effects, so that the statistical nature of the particles becomes essential. In a Boson gas, at a temperature close to the absolute zero, the wave function of the system of $N$ particles $\mathbf{x}_{1}, \ldots, \mathbf{x}_{N} \in \mathbb{R}^{3}$ may be written with the use of a single wave function $\psi$ :

$$
\tilde{\psi}\left(\mathbf{x}_{1}, \ldots, \mathbf{x}_{N}\right)=\Pi_{j=1}^{N} \psi\left(\mathbf{x}_{j}\right)
$$

Denoting by $V$ the external potential allowing to confine the system, and taking account of two-body interactions through the symmetric interaction potential $U$, the Hamiltonian of the system may be written as

$$
\widehat{H}=\sum_{j=1}^{N}\left(-\frac{\hbar^{2}}{2 m} \Delta_{\mathbf{x}_{j}}+V\left(\mathbf{x}_{j}\right)\right)+\sum_{1 \leq j, k \leq N} U\left(\mathbf{x}_{j}-\mathbf{x}_{k}\right)
$$

This induces the following expression for the energy of the system, after rescaling the wave function $\psi$ by a factor $\frac{1}{\sqrt{N}}$ and assuming $N$ is large :

$$
\mathcal{E}(\psi)=\int_{\mathbb{R}^{3}}\left[\frac{\hbar^{2}}{2 m}|\nabla \psi(t, x)|^{2}+V(x)|\psi(t, x)|^{2}+\frac{1}{2}\left(\int_{\mathbb{R}^{3}} U\left(x^{\prime}-x\right)\left|\psi\left(t, x^{\prime}\right)\right|^{2} d x^{\prime}\right)|\psi(t, x)|^{2}\right] d x
$$

and the Schrödinger evolution

$$
i \hbar \partial_{t} \psi(t, x)=\nabla_{\psi} \mathcal{E}(\psi)(t, x)
$$

Now, when the distance between the particles is less than the de Broglie wavelength $\lambda_{T}$ and for weak interactions, the particles do not see the details of the interaction potential and this later may be replaced with the effective potential $U_{\text {eff }}(x)=\frac{4 \pi \hbar^{2} a_{s}}{m} \delta_{0}(x)$. Here $a_{s}$ is the atomic diffusion length, a constant that may be positive or negative. We then finally end up with the evolution equation

$$
i \hbar \partial_{t} \psi=-\frac{\hbar}{2 m} \Delta \psi(t, x)+V(x) \psi(t, x)+\frac{4 \pi \hbar^{2} a_{s}}{m}|\psi|^{2} \psi(t, x),
$$

usually called the Gross-Pitaevskii (GP) equation (see [4,30]). One may also consult [29], for rigorous results on this derivation.

Many effects may be added in the system, which can still be handled thanks to the above mean field procedure. The effects of rotation of the condensate - which, above a certain critical speed leads to the nucleation of vortices, a signature of the superfluid behaviour - translate into the addition of a first order term in the (GP) equation (see [4]). Dipolar interations may also be taken into account, for the modeling of condensates of atoms with strong magnetic moments, like e.g. ${ }^{52} \mathrm{Cr}$, and lead to additional nonlocal terms in (GP). Multicomponents BEC are also widely studied nowadays in the mean field approximation.

Our interest here lies in the case where external fluctuations are taken into account and modeled thanks to the addition of stochastic terms. This is the case e.g. when fluctuations of the parameters of the laser used for the confinement are taken into account. It leads to a (GP) equation in which the confining potential is perturbed by a random stationary process, that can be approximated with white noise (see $[1,2,25,27,33])$. We will review in Section 1 the mathematical and numerical results on the corresponding (GP) equation.

Another situation in which stochastic effects are included in the model is the case where the temperature is close to the critical temperature of condensation. In this partially condensed situation, interactions of the condensate with the "thermal cloud" formed by non-condensed atoms need to be taken into account. Based on the remark that a consistent description of those interactions should preserve the principles of the fluctuationdissipation theorem, thus leading (formally) to the relaxation of the system to the expected physical equilibrium, both dissipation and stochastic fluctuations are included in the (GP) equation (see [6, 21, 24]). A review of 
some mathematical and numerical results on the corresponding model - so called (projected) stochastic GrossPitaevskii equation - is presented in Section 2. Note that the model has proved to be particularly efficient in reproducing the experimentally observed formation of defects in the condensation process, consisting in decreasing the gas temperature from $T$ above the critical temperature $T_{c}$ to $T<T_{c}$, while in the same time increasing the chemical potential $\mu$ (see [34], and Section 2).

\section{RANDOM VARIATIONS OF THE CONFINING POTENTIAL}

In the mean field theory of BEC based on the Gross-Pitaevskii (GP) equation, fluctuations of the laser field intensity can be regarded as modulations of the trap potential. Such a model has been considered e.g. in [1] where moments methods were used to discuss possible collapse of the (attractive) condensate, in space dimension 2 , for a radially symmetric condensate. Fluctuations were then modeled by a delta correlated Gaussian process, that is a white noise in time. The same model, but with a more general time-dependent stationary process was also considered in the three-dimensional case in [25], and considerations about the noise power spectrum may be found in [33].

In a far-off resonance trap created by red detuned lasers, one may write the effective trapping potential in the form $V(t, x)=-\frac{1}{4} \alpha|\mathcal{E}(t, x)|^{2}$, where $\alpha$ is the atomic polarizability and $\mathcal{E}(t, x)$ is the field amplitude (see [25,33]). Then, if the dynamics of the trapped atoms is described by the Hamiltonian

$$
H=\frac{p^{2}}{2 m}+\frac{1}{2} m \omega_{\mathrm{tr}}^{2}(1+\varepsilon(t)) x^{2}
$$

where $\omega_{\mathrm{tr}}=\frac{k_{0}}{m}$ is the mean-square trap oscillation frequency (which is proportional to the time-averaged laser intensity $\left.I_{0}\right)$ and $\varepsilon(t)$ represents the fluctuations in the spring constant $k$, with $\varepsilon(t)=\frac{I(t)-I_{0}}{I_{0}}$, one ends up with the Gross-Pitaevskii equation with stochastic potential

$$
i \hbar \partial_{t} \psi=-\frac{\hbar^{2}}{2 m} \Delta \psi+V(t, x) \psi+g|\psi|^{2} \psi
$$

Here, the nonlinear coefficient is $g=\frac{4 \pi \hbar^{2} a_{s}}{m}$, and in the harmonic situation described above, $V$ has the expression

$$
V(t, x)=\frac{m \omega_{\mathrm{tr}}^{2}}{2} x^{2}(1+\varepsilon(t)) .
$$

In general, the fluctuation process $\varepsilon(t)$ is taken stationary, zero mean, and with sufficiently decaying correlation function $c(t-s)=\mathbb{E}(\varepsilon(t) \varepsilon(s))$.

Introducing the variables $t^{\prime}=\omega_{\mathrm{tr}} t, x^{\prime}=\frac{x}{r_{0}}$ with $r_{0}=\sqrt{\frac{\hbar}{m \omega_{\mathrm{tr}}}}$ and $\psi^{\prime}=\sqrt{4 \pi\left|a_{s}\right| r_{0}^{2}} \psi, \varepsilon^{\prime}\left(t^{\prime}\right)=\varepsilon\left(t^{\prime} / \omega_{\mathrm{tr}}\right)$ and dropping the primes, allows to write (1) in dimensionless form

$$
i \partial_{t} \psi=-\frac{1}{2} \Delta \psi+\frac{1}{2} x^{2}(1+\varepsilon(t)) \psi+\lambda|\psi|^{2} \psi
$$

where $\lambda=\operatorname{sign}\left(a_{s}\right)= \pm 1$. A variational approach was used in [25], assuming a Gaussian profile for the wave function of the condensate of the form

$$
\psi(t, x)=A(t) \exp \left(-\frac{|x|^{2}}{2 a(t)}+\frac{i}{2} b(t)|x|^{2}+i \theta(t)\right) ;
$$

moreover, a diffusion-approximation theorem was used on the system rewritten in terms of action-angle variables, showing that the dynamics of the action variable is well approximated by a Markov diffusion process, with a generator depending on some integral in time of the correlation function $c$. This allowed the authors to predict 
the collapse time (in the attractive cas $\lambda=-1$ ) and compare it with numerical simulations. Such considerations on the collapse had also previously been considered in the two-dimensional case in [1].

From a mathematical point of view, considering a delta-correlated fluctuation process $\varepsilon$ (see also Theorem 2 below), it is convenient to write the equation as a Stratonovich stochastic partial differential equation, with the help of a real valued Brownian motion $W$ on a probability space $(\Omega, \mathcal{F}, \mathbb{P})$ endowed with a filtration $\left(\mathcal{F}_{t}\right)_{t \geq 0}$ associated with $W$ and writing formally $\varepsilon(t)=\sigma_{0} \frac{d W}{d t}$. We then find

$$
i d \psi=-\frac{1}{2}(\Delta \psi-V(x) \psi) d t+\lambda|\psi|^{2} \psi d t+\frac{\sigma_{0}}{2} V(x) \psi \circ d W,
$$

where $V(x)=|x|^{2}, \sigma_{0} \in \mathbb{R}, \lambda= \pm 1$ and $\circ$ stands for a Stratonovich product in the right hand side of (3). Note that the choice of a Stratonovich product is natural at least for two reasons : first, as explained above, this delta-correlated situation is the limiting case (for vanishing correlation length) of a more general situation where the fluctuations are modeled with a stationary process; secondly, such a noise allows the preservation of the total number of atoms, $N=|\psi|_{L^{2}}^{2}$, almost surely (see below) which is also a natural assumption.

As is classical, we may rewrite equation (3) as a Itô equation :

$$
i d \psi=-\frac{1}{2}(\Delta \psi-V(x) \psi) d t+\lambda|\psi|^{2} \psi d t+\frac{\sigma_{0}}{2} V(x) \psi d W-i \frac{\sigma_{0}^{2}}{4} V^{2}(x) \psi d t .
$$

This equation has been studied in the series of papers [15-18] and [31]. The question of interest were in particular the local and global existence of solutions (and the possible collapse, in the attractive case $\lambda=-1$, which was previously discussed in the physics papers), the dynamics of the solutions close to a multiple vortex, numerical simulations and the numerical analysis of the Crank-Nicolson scheme. We describe in more details the corresponding results in the following subsections.

\subsection{Existence results and diffusion-approximation}

Note that in equation (3), the noise term $\frac{\sigma_{0}}{2} V(x) \psi \circ d W$, although linear in $\psi$, occurs at the highest order of the operator $-\Delta+V(x)$ of the linear evolution (this was also the case for the (NLS) equation with white noise dispersion considered e.g. in $[13,19])$. A consequence is that the noise term cannot be treated as a perturbation of the deterministic equation.The strategy in [17] is rather to use the Gauge transform

$$
\psi(t, x)=e^{-i G(t, x)} u(t, x),
$$

with $G(t, x)=\frac{1}{2} V(x)(t+W(t))$. The new wave function $u$ then satisfies the following Schrödinger equation with a random magnetic field:

$$
i \partial_{t} u=-\frac{1}{2}(\nabla-i A(t, x))^{2} u+\lambda|u|^{2 \sigma} u .
$$

with $A=\nabla G(t, x)=\frac{1}{2} \nabla V(x)(t+W(t))$.

In order to obtain local existence results for equation (6), we need dispersive estimates on the linear evolution, deduced from "good" properties of the integral kernel of the linear evolution propagator (with $\lambda=0$ ), which can be expressed in terms of classical orbits, as is often used in semiclassical analysis. Using these classical paths, we can write the propagator as an oscillatory integral operator associated to the action integral. Such oscillatory integral operators have been studied by many authors in the context of deterministic Schrödinger equations (see e.g. $[22,28,35]$ ). The theory of [35] does not apply directly to equation (6), since it requires that the time derivative of the vector potential $A(t, x)$ is uniformly bounded, while this time derivative only exist in a distribution sense in our case, since $\dot{W}(t)$ is a white noise. We were however able to prove, making use of the almost sure $C^{\alpha}$ regularity of the Brownian motion, with $0<\alpha<1 / 2$, that the estimates in [35] can be generalized to our case. Actually, in our study, $W(t)$ could be replaced by any real valued $C^{\alpha}$ function of the time variable, with $\alpha>0$. The analysis was generalized in [20] to the case of more general Hamiltonian, allowing to consider the situation where rotation of the condensate is taken into account. 
Using those dispersive estimates, we obtained in [17] the following proposition concerning the local solutions of equation (6). Here $\Sigma$ denotes the energy space

$$
\Sigma=\left\{v \in L^{2}\left(\mathbb{R}^{d}\right),|\nabla v|_{L^{2}}^{2}+|x v|_{L^{2}}^{2}=|v|_{\Sigma}^{2}<+\infty\right\}
$$

Proposition 1. Assume $d=1,2$ or $3, \lambda= \pm 1$ and $r=8 / d$.

(i) Let $d=1$ and $u_{0} \in L^{2}(\mathbb{R})$. Then there exists a unique global solution $u$ of $(6)$, adapted to $\left(\mathcal{F}_{t}\right)_{t \geq 0}$, almost surely in $C\left(\left[0, T_{0}\right] ; L^{2}(\mathbb{R})\right) \cap L^{r}\left(0, T_{0} ; L^{4}(\mathbb{R})\right)$ for any $T_{0}>0$. Moreover, the $L^{2}$ norm is conserved:

$$
|u(t)|_{L^{2}}=|u(0)|_{L^{2}}, \quad \text { a.s. in } \omega, \quad \text { for all } t \geq 0
$$

and $u$ depends continuously on the initial data $u_{0}$ in the following sense: if $u_{0, n} \rightarrow u_{0}$ in $L^{2}(\mathbb{R})$, and if $u_{n}$ denotes the solution of (6) with $u_{0}$ replaced by $u_{0, n}$, then $u_{n} \rightarrow u$ in $L^{\infty}\left(0, T_{0} ; L^{2}\right)$, a.s.

(ii) Let $d=1$ and $u_{0} \in \Sigma$. Then there exists a unique global adapted solution $u$ of (6) almost surely in $C\left(\mathbb{R}^{+} ; \Sigma\right)$.

(iii) Let $u_{0} \in \Sigma$, and $d=2$ or 3 . Then there exists a maximal time $T^{*}=T_{u_{0}, \omega}^{*}>0$ such that there exists a unique adapted solution $u(t)$ of $(6)$ almost surely in $C\left(\left[0, T^{*}\right) ; \Sigma\right)$, and the following alternative holds, a.s. : $T^{*}=+\infty$ or $T^{*}<+\infty$ and $\lim _{t \uparrow T^{*}}|u(t)|_{\Sigma}=+\infty$.

We actually considered in [17] more general power nonlinearities, and the relation between the power $|u|^{2 \sigma} u$ and the space dimension $d$ for local existence in $L^{2}\left(\mathbb{R}^{d}\right)$ is the usual condition $\sigma<2 / d$. An application of the inverse of the Gauge transform given by (5) immediately leads to the corresponding local existence and uniqueness result for equation (3) (or equivalently (4)), and the preservation of the $L^{2}$-norm of $\psi$, almost surely. Global results in the energy space $\Sigma$ are deduced from the evolution of the Hamiltonian

$$
H(\psi)=\frac{1}{4}|\nabla \psi|_{L^{2}}^{2}+\frac{1}{4} \int_{\mathbb{R}^{d}} V(x)|\psi(x)|^{2} d x+\frac{\lambda}{4}|\psi|_{L^{4}}^{4}
$$

Indeed, an application of the Itô formula to equation (4) allows to show that for any stopping time $\tau \leq T^{*} \wedge T$ :

$$
H(\psi(\tau))=H\left(\psi_{0}\right)+\frac{\sigma_{0}^{2}}{16} \int_{0}^{\tau} \int_{\mathbb{R}^{d}}|\nabla V(x)|^{2}|\psi(x)|^{2} d x d t-\frac{\sigma_{0}}{4} \operatorname{Im} \int_{0}^{\tau} \int_{\mathbb{R}^{d}} \nabla V(x) \cdot \nabla \psi(x) \bar{\psi}(x) d x d W, \quad \text { a.s }
$$

and

$$
\int_{\mathbb{R}^{d}} V(x)|\psi(\tau, x)|^{2} d x=\int_{\mathbb{R}^{d}} V(x)\left|\psi_{0}\right|^{2} d x+\operatorname{Im} \int_{0}^{\tau} \int_{\mathbb{R}^{d}} x \bar{\psi} \cdot \nabla \psi d x d t, \quad \text { a.s. }
$$

The attractive case $\lambda=-1$ was already proved to possibly lead to the collapse of the wave function $\psi$ in [15] and we end up with the following existence and uniqueness results for equation (3).

Theorem 1. Assume $d=1,2$ or $3, \lambda= \pm 1$ and $r=8 / d$.

(i) Let $d=1$ and $\psi_{0} \in L^{2}(\mathbb{R})$. Then there exists a unique global solution $\psi(t)$ of $(3)$, adapted to $\left(\mathcal{F}_{t}\right)_{t \geq 0}$ with $\psi(0)=\psi_{0}$, which is almost surely in $C\left(\mathbb{R}^{+} ; L^{2}(\mathbb{R})\right) \cap L_{\text {loc }}^{r}\left(\mathbb{R}^{+} ; L^{4}\right)$. Moreover, the $L^{2}$-norm is conserved by the time evolution, that is,

$$
\left|\psi_{0}\right|_{L^{2}}=|\psi(t)|_{L^{2}}, \quad \text { a.s., for all } t \geq 0
$$

If moreover $\psi_{0} \in \Sigma$, then $\psi$ is almost surely in $C\left(\mathbb{R}^{+} ; \Sigma\right)$.

(ii) Let $d=2$ or 3 , and $\psi_{0} \in \Sigma$. Then there exist a stopping time $\tau^{*}=\tau_{\psi_{0}}^{*}>0$ and a unique solution $\psi(t)$ of $(3)$, adapted to $\left(\mathcal{F}_{t}\right)_{t \geq 0}$ with $\psi(0)=\psi_{0}$, almost surely in $C\left(\left[0, \tau^{*}\right) ; \Sigma\right)$. In fact, $\tau^{*}=T^{*}$, defined in 
Proposition 1 (iii). If $\lambda=1$, then $T^{*}=+\infty$. If $\lambda=-1$ and there exists a deterministic time $\bar{t}>0$ such that

$$
\int_{\mathbb{R}^{d}} V(x)\left|\psi_{0}(x)\right|^{2} d x+4 \bar{t} \operatorname{Im} \int_{\mathbb{R}^{d}} \bar{\psi}_{0}(x) x . \nabla \psi_{0}(x) d x+8 H\left(\psi_{0}\right) \bar{t}^{2}<0,
$$

then $\mathbb{P}\left(T_{\psi_{0}}^{*} \leq \bar{t}\right)>0$, i.e. collapse occurs with a positive probability.

As a byproduct of the representation formula, and in particular of the continuous dependence of the solution $u$ given by Proposition 1 with respect to the trajectories of the Brownian motion, that can be proved thanks to this representation formula in the $L^{2}$-subcritical case (which implies $d=1$ ), a diffusion-approximation result was obtained in [17], which justifies in particular the choice of time white noise in some scaling. Indeed, consider a process $m(t)$ which is either a stationary ergodic process satisfying some mixing conditions, or a Markov process with a unique ergodic invariant measure, so that by the Donsker functional central limit theorem, the process $t \mapsto \frac{\varepsilon}{\sigma_{0}} \int_{0}^{t / \varepsilon^{2}} m(s) d s$ converges in distribution in $C([0, T])$ to a standard real valued Brownian motion. Then the following holds.

Theorem 2. Let $d=1, \lambda= \pm 1$ and $(m(t))_{t \geq 0}$ as above. Then, for any $\varepsilon>0$ and $\psi_{0} \in L^{2}(\mathbb{R})$ there exists a unique solution $\varphi_{\varepsilon}$, with continuous paths on $\mathbb{R}^{+}$with values in $L^{2}(\mathbb{R})$, of the following equation:

$$
\left\{\begin{array}{l}
i \partial_{t} \varphi=\frac{1}{2}(-\Delta+V(x)) \varphi+\lambda|\varphi|^{2} \varphi+\frac{1}{2 \varepsilon} m\left(\frac{t}{\varepsilon^{2}}\right) V(x) \varphi \\
\varphi(0)=\psi_{0} .
\end{array}\right.
$$

Moreover the process $\varphi_{\varepsilon}$ converges in distribution in $C\left([0, T] ; L^{2}\left(\mathbb{R}^{d}\right)\right)$ as $\varepsilon$ tends to zero, to the solution $\psi$ of (3), for any positive $T$.

\subsection{Dynamics of solutions close to a multiple vortex}

In dimension two, some specific solutions of the deterministic equation and for repulsive condensates, that is Equation (3) with $\sigma_{0}=0$ and $\lambda=1$, are given by vortices, which are solutions of the form

$$
u(t, r, \theta)=e^{-i \mu t} e^{i m \theta} \psi_{\mu, m}(r)
$$

where $r, \theta$ are polar coordinates, $m \in \mathbb{N}$ is the vortex degree, $\mu$ is the chemical potential and $\psi_{\mu, m}(r)$ is the radial positive vortex profile. We show in figures 1 and 2 some profiles $\psi_{\mu, m}$, for different values of $m$ and $\mu$.

The influence of white noise perturbations of the confining potential on the dynamics of those solutions has been studied in [18] (and in [16] in the case $m=0$ ), in the small noise asymptotics. More precisely, consider the following stochastic Gross-Pitaevskii equation

$$
i d u+\left(\Delta u-|x|^{2} u-|u|^{2 \sigma} u\right) d t=\varepsilon|x|^{2} u \circ d W,
$$

where $\circ$ stands for a Stratonovich product in the right hand side of (12), $\sigma>0, \varepsilon>0$. Here, as before, $W(t)$ is a standard real valued Brownian motion on $\mathbb{R}^{+}$. We recall that $\Sigma$ is the energy space consisting of square integrable functions $f$ such that $\nabla f \in L^{2}\left(\mathbb{R}^{2}\right)$ and $x f \in L^{2}\left(\mathbb{R}^{2}\right)$. Let then $X_{m}$ be the closed subspace of $\Sigma$ that has the same symmetry, i.e., $m$-equivariant symmetry as the vortex solutions $\phi_{\mu, m}(x)=e^{i m \theta} \psi_{\mu, m}(r)$ :

$$
X_{m}:=\left\{v \in \Sigma, \quad e^{-i m \theta} v(x) \text { does not depend on } \theta\right\} .
$$

One may check (see [18] for details) that the noise in the right hand side of (12) preserves the equivariance structure, or in other terms that the solution $u(t)$ of (12) belongs to $X_{m}$ if the initial data $u(0)=u_{0}$ belongs to $X_{m}$. Defining then the action functional $S_{\mu}$ as

$$
S_{\mu}(u)=\frac{1}{2}|\nabla u|_{L^{2}}^{2}+\frac{1}{2}|x u|_{L^{2}}^{2}+\frac{1}{2 \sigma+2}|u|_{L^{2 \sigma+2}}^{2 \sigma+2}-\frac{\mu}{2}|u|_{L^{2}}^{2},
$$




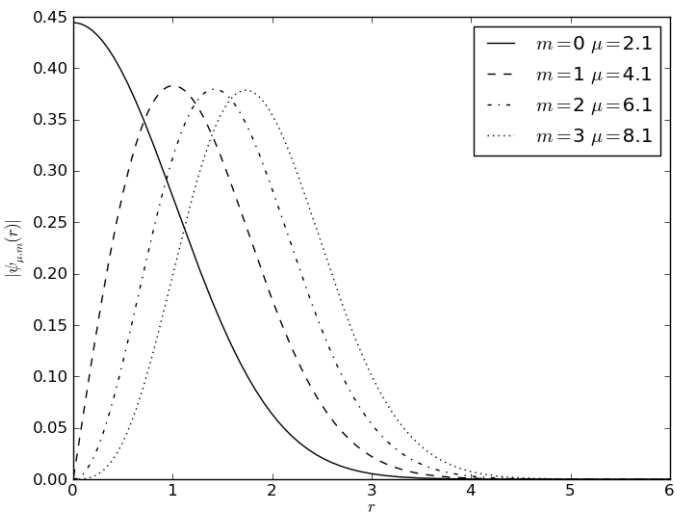

FIGURE 1. Amplitudes of vortices $\left|\psi_{\mu, m}\right|$ for $\mu$ close to $\lambda_{m}$, for different

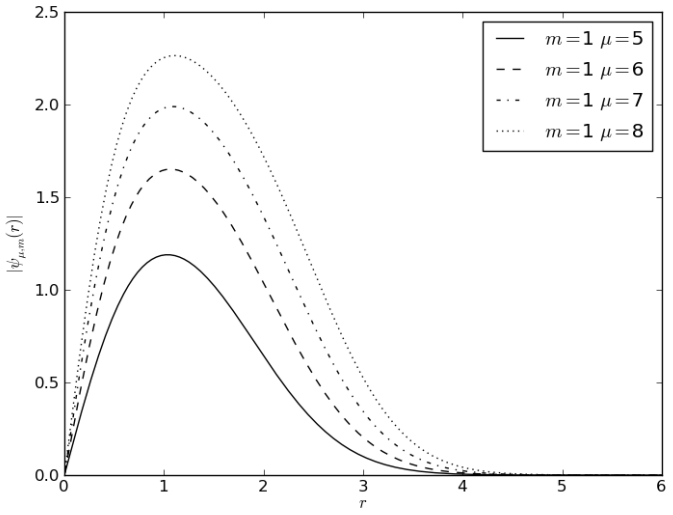

FIGURE 2. Amplitudes of vortices $\left|\psi_{\mu, m}\right|$ for $m=1$ for different values of $\mu$

which is a conserved quantity of the deterministic equation, the profile $\phi_{\mu, m}=e^{i m \theta} \psi_{\mu, m}(r)$ of the vortex solution (11) may be characterized as the unique global minimizer of $S_{\mu}$ on $X_{m}$, as soon as $\mu>\lambda_{m}=2 m+1$. A straightforward consequence of this is the stability of the vortex solution (11) for the deterministic equation (12) with $\varepsilon=0$.

Using this stability property, the following result was proved in [18] concerning the solution $u^{\varepsilon}(t, x)$ of equation (12) with initial data $u^{\varepsilon}(0, x)=\phi_{\mu_{0}, m}(x)$ : it says that we can decompose $u^{\varepsilon}$ in $X_{m}$ as the sum of a modulated vortex solution and a remainder with small $\Sigma$ norm, for $t$ less than some stopping time $\tau^{\varepsilon}$, and that this $\tau^{\varepsilon}$ goes to infinity in probability as $\varepsilon$ goes to zero. This decomposition is in the form

$$
u^{\varepsilon}(t, x)=e^{-i \xi^{\varepsilon}(t)}\left(\phi_{\mu_{0}, m}(x)+\varepsilon \eta^{\varepsilon}(t, x)\right)
$$

for a semi-martingale process $\xi^{\varepsilon}(t)$ with values in $\mathbb{R}$, and $\eta^{\varepsilon}$ with value in $X_{m}$. Here, we use the following orthogonality condition

$$
\operatorname{Re}\left(\eta^{\varepsilon}, i \phi_{\mu_{0}, m}\right)_{L^{2}\left(\mathbb{R}^{2}\right)}=0, \quad \text { a.s., } \quad t \leq \tau^{\varepsilon},
$$

for a suitable stopping time $\tau^{\varepsilon}$, which corresponds to the exit time of $\varepsilon \eta^{\varepsilon}$ from a small neighborhood of 0 in $\Sigma$. More precisely, we have the following result.

Theorem 3. Let $1 / 2 \leq \sigma<\infty$ and $\mu_{0}>\lambda_{m}$ be fixed. For $\varepsilon>0$, let $u^{\varepsilon}(t, x)$ be the solution of (12) with $u(0, x)=\phi_{\mu_{0}, m}(x)$. Then there exists $\alpha_{0}>0$ such that, for each $\alpha, 0<\alpha \leq \alpha_{0}$, there is a stopping time $\tau_{\alpha}^{\varepsilon} \in(0, \infty)$ a.s., and there is a semi-martingale process $\xi^{\varepsilon}(t)$, defined a.s. for $t \leq \tau_{\alpha}^{\varepsilon}$, with values in $\mathbb{R}$, so that if we set $\varepsilon \eta^{\varepsilon}(t, x)=e^{i \xi^{\varepsilon}(t)} u^{\varepsilon}(t, x)-\phi_{\mu_{0}, m}(x)$, then (15) holds. Moreover, a.s. for $t \leq \tau_{\alpha}^{\varepsilon}$,

$$
\left|\varepsilon \eta^{\varepsilon}(t)\right|_{\Sigma} \leq \alpha .
$$

In addition, there is a constant $C=C_{\alpha, \mu_{0}, m}>0$, such that for any $T>0$ and any $\alpha \leq \alpha_{0}$, there is an $\varepsilon_{0}>0$, such that for each $\varepsilon<\varepsilon_{0}$,

$$
\mathbb{P}\left(\tau_{\alpha}^{\varepsilon} \leq T\right) \leq \exp \left(-\frac{C}{\varepsilon^{2} T}\right) .
$$

The modulation parameter $\xi^{\varepsilon}$ may be written, for $t \leq \tau_{\alpha}^{\varepsilon}$, as

$$
d \xi^{\varepsilon}=\mu_{0} d t+\varepsilon y^{\varepsilon} d t+\varepsilon z^{\varepsilon} d W
$$


for some adapted processes $y^{\varepsilon}$, $z^{\varepsilon}$ with values in $\mathbb{R}$ satisfying: as $\varepsilon$ goes to zero, $y^{\varepsilon}, z^{\varepsilon}$ converge respectively to some real valued processes $y, z$ in probability in $C([0, T])$.

Numerical simulations were also performed in [18], which showed numerical evidences of the optimality of the estimate (17), in terms of rates in time and $\varepsilon$. Taking into account the $m$-equivariant symmetry, Equation (12) is only solved in the radial component and reduces to a one dimensional equation. Numerical simulations were performed with a numerical scheme based on a Crank-Nicolson discretization in time, which is natural to consistently approximate the Stratonovich integral (see also section 1.3 below). The nonlinearity is not computed by a fixed point method, but by a relaxation method which amounts to compute an explicit extrapolation of the nonlinearity. Note that this later method does not preserve exactly the L2 norm, but with a small enough space discretization, the numerical simulations showed negligible fluctuations. The space approximation is based on finite differences. The radial profile of the vortex solutions (which are used as Cauchy condition) were computed by a shooting method. Figure 3 and 4 display respectively the time evolution of the radial profile of a solution,

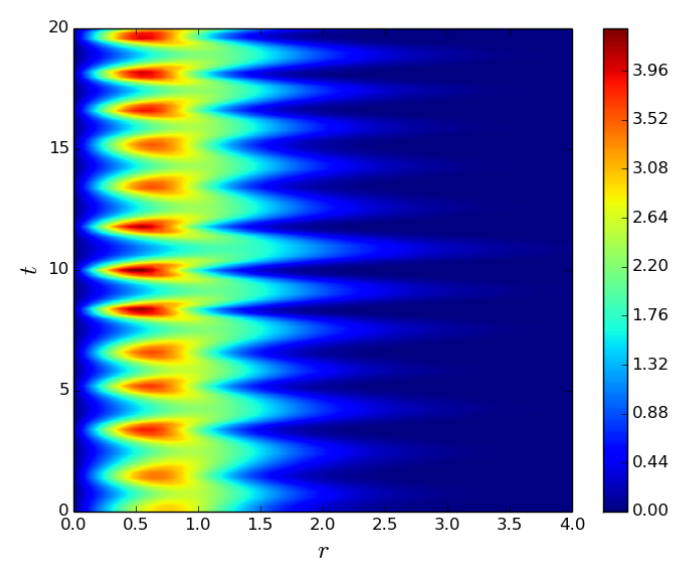

FiguRE 3. A trajectory of $\left|u^{\epsilon}(r, t)\right|$ for $m=1$ and $\sigma=1$

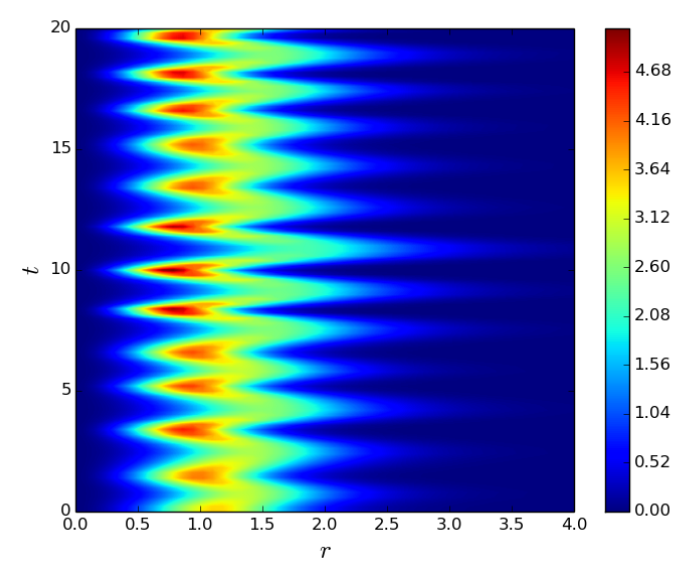

Figure 4. A trajectory of $\left|u^{\epsilon}(r, t)\right|$ for $m=2$ and $\sigma=1$

taking as initial Cauchy condition a vortex solution with vortex degree $m=1$ and $m=2$. We can observe that the wave function keeps the same structure over time and that it oscillates with an almost periodic rhythm.

The computation of the processes $\xi^{\varepsilon}(t)$ and $\eta^{\varepsilon}(t, x)$ are straightforward using the orthogonality condition (15) and are given almost surely for $t \leq \tau^{\varepsilon}$ by,

$$
\xi^{\varepsilon}(t)=-\arg \left(u^{\varepsilon}(t), \phi_{\mu_{0}, m}\right)_{L^{2}\left(\mathbb{R}^{2}\right)}, \quad \text { and } \quad \varepsilon \eta^{\varepsilon}(t, x)=u^{\varepsilon}(t, x) e^{i \xi^{\varepsilon}(t)}-\phi_{\mu_{0}, m}(x) .
$$

An approximation of the law of the stopping time $\tau_{\alpha}^{\varepsilon}$ with $\varepsilon=9 \cdot 10^{-3}$ was computed using a classical MonteCarlo method, and is given in Figure 5 and 6 . We can observe that the slopes therein (for $\varepsilon$ small enough) are in good agreement with estimate (17).

\subsection{Numerical analysis of the Crank-Nicolson scheme}

The use of a Crank-Nicolson scheme to discretize equation (3) is natural since mid-point discretization is consistent with the Stratonovich product. It also preserves the conservation of $L^{2}$ norm. Such a discretization has been used in $[5,12,26]$ for other types of stochastic nonlinear Schrödinger equations. Even if, in terms of computational costs, it is often convenient to consider its linearly implicit derivative given by the relaxation scheme (see the above simulations), the numerical analysis of this later scheme is closely related to the numerical 


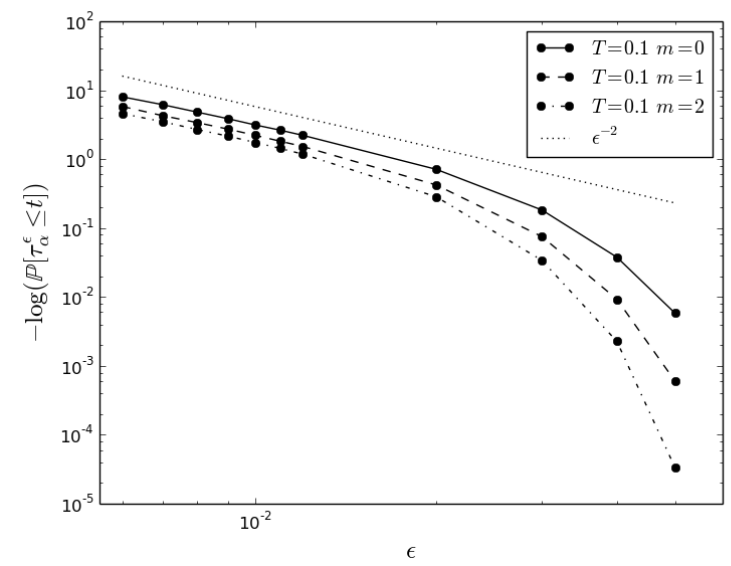

FiguRE 5. Estimation of $\mathbb{P}\left(\tau_{\alpha}^{\varepsilon} \leq t\right)$ by a Monte-Carlo method, with respect to $\epsilon$

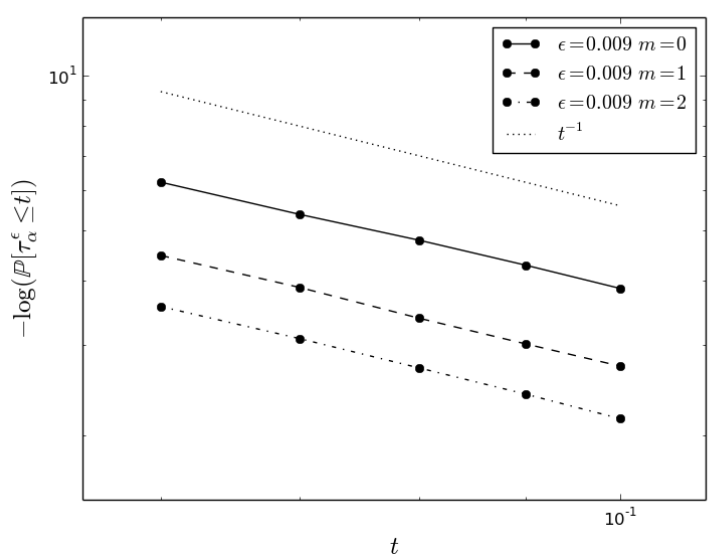

FiguRe 6. Estimation of $\mathbb{P}\left(\tau_{\alpha}^{\varepsilon} \leq t\right)$ by a Monte-Carlo method, with respect to $t$

analysis of the Crank-Nicolson scheme, which is in some sense a prerequisite. This numerical analysis has been carried out in [31], where the convergence at order one of the time-semi-discrete scheme was proved.

The naive Crank-Nicolson discretization of (3) is given by

$$
i \frac{\psi_{n+1}-\psi_{n}}{\delta t}=\frac{1}{2}\left(A+\frac{\chi_{n+1}}{\sqrt{\delta t}}|x|^{2}\right)\left(\frac{\psi_{n}+\psi_{n+1}}{2}\right)+\lambda g\left(\psi_{n}, \psi_{n+1}\right),
$$

where $A=-\Delta+|x|^{2}, \psi_{n}$ is an approximation of $\psi(n \delta t),\left(\chi_{n}\right)_{n \geq 0}$ is a sequence of independent normal random variables and $g$ is the approximation of the nonlinearity given by

$$
g\left(\psi_{n}, \psi_{n+1}\right)=\frac{1}{2}\left(\left|\psi_{n}\right|^{2}+\left|\psi_{n+1}\right|^{2}\right)\left(\frac{\psi_{n}+\psi_{n+1}}{2}\right) .
$$

There are several difficulties in this analysis. The first one is the non Lipschitz behavior of the nonlinearity, which is in particular an obstacle to the global existence and uniqueness of a solution $\psi_{n}$ of (18), and requires the use of a cut-off for the nonlinear term. Secondly, although the above scheme allows the conservation of the $L^{2}$ norm, giving immediately the $L^{2}$-stability, it does not allow to get stable estimates on higher order derivatives, which are needed to study the order of convergence of the scheme. The argument is again to use a cut-off in the highest modes of the operator $A=-\Delta+|x|^{2}$ which would correspond to the use of a spectral space discretization. Note that a different argument was used in [9], for the standard, cubic nonlinear Schrödinger equation with multiplicative noise, consisting in slightly more impliciteness in the scheme, and leading to better stability properties. However, the argument does not apply in the present situation, were the lack of stability is due to a lack of "good" commutator properties between the noise and the linear operator.

The result may be stated as follows. Denote, for $k \in \mathbb{N}$,

$$
\Sigma^{k}=\left\{v \in L^{2}\left(\mathbb{R}^{d}\right), \sum_{|\alpha|+|\beta| \leq k}\left|x^{\beta} \partial_{x}^{\alpha} v\right|_{L^{2}}^{2}:=|v|_{\Sigma^{k}}^{2}<+\infty\right\}
$$

We denote by $\psi$ the solution of (3) with $\psi(0)=\psi_{0}$ and we consider, for a fixed time step $\delta t$ a solution $\left(\psi_{K, L, \delta t}^{n}\right)_{n \delta t \leq T}$ of (18) where $A$ is replaced by $A_{k}=P_{K} A, P_{K}$ being the projection on the $K$ first eigenmodes 
of $A$, and $g$ is replaced by a Lipschitz approximation with Lipschitz constant $L$ (see [31] for details), and with $\psi_{K, L, \delta t}^{n}(0)=\psi_{0}$. Then the following convergence result holds.

Theorem 4. For all $T>0, k \in \mathbb{N}, \psi_{0} \in \Sigma^{k+12}\left(\mathbb{R}^{d}\right), C>0$ and $\alpha<1$, there is a choice of the cut-off constants $K(\delta t), L(\delta t)$ such that

$$
\lim _{\delta t \rightarrow 0} \sup _{n \delta t \leq T} \mathbb{P}\left[\left|\psi_{K(\delta t), L(\delta t), \delta t}^{n}-\psi\left(t_{n}\right)\right|_{\Sigma^{k}} \geq C(\delta t)^{\alpha}\right]=0 .
$$

\section{A model With Positive temperature : the Projected stochastic Gross-PitAevskiI EQUATION}

When temperature is close to the critical temperature of condensation, one has to take into account the non-condensate particle contributions, which are negligible for temperature close to the absolute zero. Even though a unified approach does not seem to be yet available in this situation, the use of a "truncated Wigner theory" (see $[6,23,24]$ ), leads to a model for the wave function which, in a simplified form taking account only of the growth terms, may be written as

$$
d \psi=\mathcal{P}_{c}\left[-\frac{i}{\hbar} L_{G P} \psi d t+\frac{\gamma}{k_{B} T}\left(\mu-L_{G P}\right) \psi d t+d W_{\gamma}(t, x)\right]
$$

where

$$
L_{G P}=-\frac{\hbar^{2}}{2 m} \Delta+V(x)+g|\psi(t, x)|^{2},
$$

$m$ is the atomic mass, $g=\frac{4 \pi \hbar^{2} a}{m}$ with $a$ the (here positive) s-wave scattering length, $V(x)=\frac{m}{2} \omega^{2} x^{2}, \mu$ is the chemical potential, and $\mathcal{P}_{c}$ is a projection operator on the lowest energy modes (the non-condensate band). Moreover, the noise term $d W_{\gamma}$ corresponds here to a complex-valued space-time white noise, that is a Gaussian field which is $\delta$-correlated both in space and time :

$$
\mathbb{E}\left(d W_{\gamma}^{*}(t, x) d W_{\gamma}\left(t^{\prime}, x^{\prime}\right)\right)=2 \gamma \delta\left(t-t^{\prime}\right) \delta\left(x-x^{\prime}\right) d t .
$$

The methodology in [24] consisted in first considering that the modes with wavenumbers $\mathbf{k}$ larger than $\Delta$, where $2 \pi / \Delta$ is the order of magnitude of the range of the interatomic potential, have no occupation and can be eliminated. Next, the remaining components of the wave function can be divided into a low momentum component (the condensate band) which is treated fully quantum-mechanically, and a high momentum component (the non-condensate band) which is considered to be a bath of thermalized atoms.

The same model was obtained previously in [21] with different methods, in particular a functional integral formulation of the Keldysh method, in which the elimination of the highest energy modes is achieved in the action functional. Moreover, the model is in very good qualitative agreement with the experiments aiming to simulate the dynamics of the condensation process (see [34], and the simulations below).

At zero temperature, one recovers the standard (GP) equation for the projected wave function $\psi_{c}$ on the lowest energy modes, for which the hamiltonian

$$
H\left(\psi_{c}\right)=\frac{\hbar^{2}}{2 m}\left|\nabla \psi_{c}\right|_{L^{2}}^{2}+\frac{m}{2}\left|\omega x \psi_{c}\right|_{L^{2}}^{2}-\mu\left|\psi_{c}\right|_{L^{2}}^{2}+\frac{g}{4}\left|\psi_{c}\right|_{L^{4}}^{4}
$$

is conserved. Note that a symmetry breaking occurs for the ground state of $H$ at $\mu=\hbar \omega$, since for small values of $\mu$ the minimum of $H$ is equal to zero. At positive temperature, it may easily be formally shown that the Gibbs measure given by

$$
\pi_{T}\left(d \psi_{c}\right)=\Gamma_{c} \exp \left(-\frac{H\left(\psi_{c}\right)}{k_{B} T}\right) d \psi_{c},
$$

for some normalizing constant $\Gamma_{c}$, is invariant under the transition semi-group associated with (19), and the convergence to equilibrium of $\psi_{c}$ with a geometric rate may be proved (see [32]). 

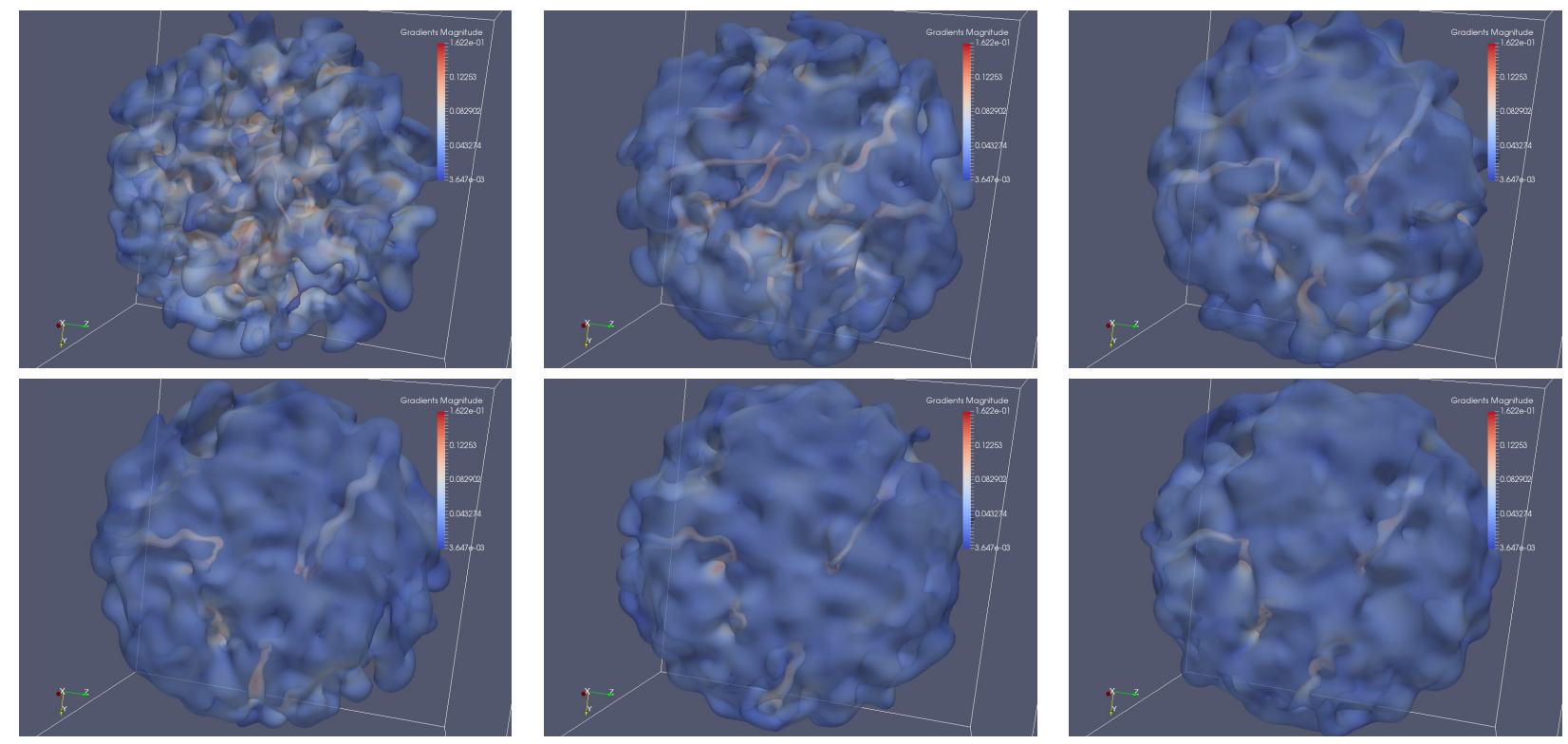

FiguRe 7. Time evolution of a BEC submitted to an instantaneous quench from $\mu=1$ to $\mu=25$ at initial time $t=0$.

This model has been used to observe the spontaneous nucleation of vortices during the condensation process (see [34]). Indeed, the appearance of topological defects during a phase transition is predicted by the KibbleZurek mechanism, and can be modeled by Equation (19) in the case of Bose-Einstein condensation. In this model, the phase transition is initiated by a positive value of the chemical potential $\mu$. Thus, starting from a thermalized state with low chemical potential and high temperature, a sudden quench to a higher chemical potential and a lower temperature will lead to the formation of vortices during the growth process of the condensate. This dynamics is shown in Figure 2. We can observe a fast appearance of vortices after the instantaneous quench, followed by a slow decay of the vortices.

The numerical scheme used to solve equation (19) is an implicit/explicit scheme for the time discretization, and a Hermite spectral discretization in space, which is natural to take precisely into account the cut-off operator $\mathcal{P}_{c}$.

From a mathematical point of view, the role of the cut-off operator $\mathcal{P}_{c}$ is also crucial as equation (19) is not expected to possess solutions if $\mathcal{P}_{c}$ is equal to the identity operator, in space dimension two or three. However, the equation has been studied in space dimension one, without the cut-off operator, in [14] and the existence of a global solution of the equation, for a.s. all initial data with respect to the invariant measure of the standard (GP) equation was proved. This later invariant measure was indeed studied in [8] and its support was shown to contain $L^{p}(\mathbb{R})$ for any $p$ larger than 2 . The convergence to equilibrium was also obtained in [14], thanks to a Poincaré inequality.

More precisely, consider the equation in dimensionless form

$$
d \psi=(i+\gamma)\left[\Delta \psi-x^{2} \psi+\mu \psi-|\psi|^{2} \psi\right] d t+\sqrt{\gamma} d W
$$

where $\psi(t, x)$ is the wave function, $x \in \mathbb{R}, \gamma>0, \mu \geq 0$, and $W$ is a cylindrical Wiener process on $L^{2}(\mathbb{R})$ that may be modeled as follows : let $\left(h_{n}\right)_{n \in \mathbb{N}}$ be a real valued complete orthonormal system in $L^{2}(\mathbb{R})$ such that $\left(-\Delta+x^{2}\right) h_{n}=\lambda_{n} h_{n}$, with $\lambda_{n}=2 n+1$, so that $\left(h_{n}\right)_{n}$ is a sequence of Hermite functions; let $\left(\beta_{n}\right)_{n \in \mathbb{N}}$ be a sequence of independent normalized complex-valued Brownian motions, and write $W(t, x)=\sum_{k \in \mathbb{N}} \beta_{k}(t) h_{k}(x)$. 
Then denoting

$$
S(\psi)=\frac{1}{2} \int_{\mathbb{R}}\left(|\nabla \psi|^{2}+|x \psi|^{2}\right) d x-\frac{\mu}{2} \int_{\mathbb{R}}|\psi|^{2} d x+\frac{1}{4} \int_{\mathbb{R}}|\psi|^{4} d x
$$

the Gibbs measure may now be formally defined by $\rho(d \psi)=e^{-S(\psi)} d \psi$. It may actually be rigorously defined - at least for $\mu=0$ - as an absolutely continuous measure with respect to the Gaussian measure on $L^{p}(\mathbb{R})$ for $p>2$, defined as the law of the random function series

$$
\varphi(\omega, x)=\sum_{n=0}^{\infty} \frac{\sqrt{2}}{\sqrt{\lambda_{n}}} g_{n}(\omega) h_{n}(x),
$$

where $\left(g_{n}\right)_{n}$ is a sequence of independent complex valued $\mathcal{N}_{\mathbb{C}}(0,1)$. Indeed, the series converges in $L^{p}(\mathbb{R})$ for any $p>2$ (see $[8]$ ). Let $\nu(d \psi)$ denote the Gaussian measure defined by the law of $\varphi$ in $L^{p}(\mathbb{R}), p>2$ (which is also the invariant measure of the flow corresponding to the linear evolution in $(20)$ for $\mu=0$ ). We may then define the Gibbs measure $\rho$ as

$$
\rho(d \psi)=\Gamma^{-1} e^{-\frac{1}{4}|\psi|_{L^{4}}^{4}} \nu(d \psi)
$$

for some normalizing constant $\Gamma$. For $\mu>0$, the rigorous definition of the measure $\rho$ requires a more subtle decomposition (see [14]).

We can then solve equation (20) locally in time in $L^{p}(\mathbb{R})$, and use the invariance of $\rho$ for the evolution to prove the following theorem.

Theorem 5. Let $\gamma>0, \mu \geq 0$ and $p \geq 3$. There exists a $\rho$-measurable set $\mathcal{O} \subset L^{p}(\mathbb{R})$ such that $\rho(\mathcal{O})=1$, and such that for $\psi_{0} \in \mathcal{O}$ there exists a unique solution of $(20), \psi(\cdot) \in C\left([0, \infty), L^{p}(\mathbb{R})\right)$ a.s., such that $\psi(0)=\psi_{0}$.

Let $\left(P_{t}\right)_{t \geq 0}$ be the transition semi-group associated with Equation (20) and defined by $P_{t} \Phi\left(\psi_{0}\right)=\mathbb{E}\left(\Phi\left(\psi\left(t, \psi_{0}\right)\right)\right)$, where $\psi\left(\cdot, \psi_{0}\right)$ is the solution of $(20)$ with $\psi(0)=\psi_{0}$, so that $P_{t}$ is well defined in $L^{2}\left(L^{p}, d \rho\right)$, for any $p \geq 3$, and any $t>0$. The following convergence result holds.

Theorem 6. Let $\gamma>0, \mu \geq 0$ and $p \geq 3$. Let $\Phi \in L^{2}\left(\left(L^{p}, d \rho\right) ; \mathbb{R}\right)$, and $\bar{\Phi}=\int_{L^{p}} \Phi(y) \rho(d y)$. Then $u(t, \cdot):=$ $P_{t} \Phi(\cdot)$ converges exponentially to $\bar{\Phi}$ in $L^{2}\left(\left(L^{p}, d \rho\right), \mathbb{R}\right)$, as $t \rightarrow \infty$; more precisely,

$$
\int_{L^{p}}|u(t, y)-\bar{\Phi}|^{2} \rho(d y) \leq e^{-\gamma t} \int_{L^{p}}|\Phi(y)-\bar{\Phi}|^{2} \rho(d y) .
$$

Note that the results of Theorem 5 and Theorem 6 are still true for more general power nonlinearities $|\psi|^{2 \sigma} \psi$ if $p \geq 2 \sigma+1$ and $p>2$. Those results represent a first step in the understanding of Equation (20). The main difference with the more classical Ginzburg-Landau equation (without the confining potential, possibly posed on a bounded domain $D$ ) is the growth of the eigenvalues $\lambda_{n}$ which makes the problem critical, even though the space dimension is only one. The study of larger dimensions, as well as the qualitative study of the dynamics, will require a more refined analysis.

\section{REFERENCES}

[1] F. K. Abdullaev, B. Baizakov, and V. Konotop, Dynamics of a bose-einstein condensate in optical trap, in Nonlinearity and Disorder: Theory and Applications, Springer, 2001, pp. 69-78.

[2] F. K. Abdullaev, J. Bronski, and R. Galimzyanov, Dynamics of a trapped 2d bose-einstein condensate with periodically and randomly varying atomic scattering length, Physica D: Nonlinear Phenomena, 184 (2003), pp. 319-332.

[3] M. H. Anderson, J. R. Ensher, M. R. Matthews, C. E. Wieman, And E. A. Cornell, Observation of bose-einstein condensation in a dilute atomic vapor, science, 269 (1995), p. 198.

[4] X. Antoine And R. DuboscQ, Modeling and computation of Bose-Einstein condensates: stationary states, nucleation, dynamics, stochasticity, in Nonlinear optical and atomic systems, vol. 2146 of Lecture Notes in Math., Springer, Cham, 2015, pp. 49-145.

[5] R. Belaouar, A. De Bouard, and A. Debussche, Numerical analysis of the nonlinear schrödinger equation with white noise dispersion, Stochastic Partial Differential Equations: Analysis and Computations, 3 (2015), pp. 103-132. 
[6] P. Blakie, A. Bradley, M. Davis, R. Ballagh, and C. Gardiner, Dynamics and statistical mechanics of ultra-cold bose gases using c-field techniques, Advances in Physics, 57 (2008), pp. 363-455.

[7] C. C. Bradley, C. Sackett, J. Tollett, and R. G. Hulet, Evidence of bose-einstein condensation in an atomic gas with attractive interactions, Physical Review Letters, 75 (1995), p. 1687.

[8] N. Burq, L. Thomann, And N. Tzvetkov, Long time dynamics for the one dimensional non linear schrödinger equation, in Annales de l'Institut Fourier, vol. 63, 2013, pp. 2137-2198.

[9] C. Chen, J. Hong, And A. Prohl, Convergence of a $\theta$-scheme to solve the stochastic nonlinear schrödinger equation with stratonovich noise, Stochastics and Partial Differential Equations: Analysis and Computations, 4 (2016), pp. $274-318$.

[10] F. Dalfovo, S. Giorgini, L. P. Pitaevskit, And S. Stringari, Theory of bose-einstein condensation in trapped gases, Reviews of Modern Physics, 71 (1999), p. 463.

[11] K. B. Davis, M.-O. Mewes, M. R. Andrews, N. Van Druten, D. Durfee, D. Kurn, and W. Ketterle, Bose-einstein condensation in a gas of sodium atoms, Physical review letters, 75 (1995), p. 3969.

[12] A. De Bound And A. Debussche, A semi-discrete scheme for the stochastic nonlinear schrödinger equation, Numerische Mathematik, 96 (2004), pp. 733-770.

[13] — The nonlinear schrödinger equation with white noise dispersion, Journal of Functional Analysis, 259 (2010), pp. 13001321.

[14] A. De Bound, A. Debussche, And R. Fukuizumi, Long time behavior of a gross-pitaevskii equation at positive temperature. Preprint.

[15] A. De Bouard And R. Fukuizumi, Stochastic fluctuations in the gross-pitaevskii equation, Nonlinearity, 20 (2007), p. 2823.

[16] _ - Modulation analysis for a stochastic nls equation arising in bose-einstein condensation, Asymptotic Analysis, 63 (2009), pp. 189-235.

[17] — Representation formula for stochastic schrödinger evolution equations and applications, Nonlinearity, 25 (2012), p. 2993.

[18] A. De Boundd, R. Fukuizumi, and R. Poncet, Vortex solutions in bose-einstein condensation under a trapping potential varying randomly in time, Discrete \& Continuous Dynamical Systems-Series B, 204 (2015).

[19] A. Debussche And Y. Tsutsumi, 1d quintic nonlinear schrödinger equation with white noise dispersion, Journal de mathématiques pures et appliquées, 96 (2011), pp. 363-376.

[20] R. DuboscQ, A construction of the fundamental solution of the schrödinger equation with a perturbed quadratic hamiltonian, Nonlinearity, 29 (2016), p. 566.

[21] R. Duine And H. Stoof, Stochastic dynamics of a trapped bose-einstein condensate, Physical Review A, 65 (2001), p. 013603.

[22] D. Fujiwara, A construction of the fundamental solution for the schrödinger equation, Journal d'Analyse Mathématique, 35 (1979), pp. 41-96.

[23] C. Gardiner, J. Anglin, And T. Fudge, The stochastic gross-pitaevskii equation, Journal of Physics B: Atomic, Molecular and Optical Physics, 35 (2002), p. 1555.

[24] C. W. Gardiner and M. J. Davis, The stochastic gross-pitaevskii equation: Ii, Journal of Physics B: Atomic, Molecular and Optical Physics, 36 (2003), p. 4731.

[25] J. Garnier, F. K. Abdullaev, and B. Baizakov, Collapse of a bose-einstein condensate induced by fluctuations of the laser intensity, Physical Review A, 69 (2004), p. 053607.

[26] M. Gazeau, Probability and pathwise order of convergence of a semidiscrete scheme for the stochastic manakov equation, SIAM Journal on Numerical Analysis, 52 (2014), pp. 533-553.

[27] M. Gehm, K. Ohara, T. Savard, and J. Thomas, Dynamics of noise-induced heating in atom traps, Physical Review A, 58 (1998), p. 3914.

[28] N. Kumano-Go, Feynman path integrals as analysis on path space by time slicing approximation, Bulletin des sciences mathematiques, 128 (2004), pp. 197-251.

[29] M. Lewin, Mean-field limit of bose systems: rigorous results, arXiv preprint arXiv:1510.04407, (2015).

[30] C. J. Pethick And H. Smith, Bose-Einstein condensation in dilute gases, Cambridge university press, 2002.

[31] R. Poncet, Numerical analysis of the Gross-Pitaevskii Equation with a randomly varying potential in time, ArXiv e-prints, (2017).

[32] G. O. Roberts And R. L. Tweedie, Exponential convergence of langevin distributions and their discrete approximations, Bernoulli, (1996), pp. 341-363.

[33] T. SAvard, K. O'HARA, AND J. Thomas, Laser-noise-induced heating in far-off resonance optical traps, Physical Review A, 56 (1997), p. R1095.

[34] C. N. Weiler, T. W. Neely, D. R. Scherer, A. S. Bradley, M. J. Davis, and B. P. Anderson, Spontaneous vortices in the formation of bose-einstein condensates, Nature, 455 (2008), pp. 948-951.

[35] K. YAJIMA, Schrödinger evolution equations with magnetic fields, Journal d'Analyse Mathématique, 56 (1991), pp. 29-76. 